\title{
A note on the exponential inequality for negatively associated random variables
}

\author{
Yu Miao Jian-Yong Mu
}

\begin{abstract}
In this paper, we establish an exponential inequality for negatively associated random variables, which improves known results. These results concern in particular the previous work of Kim and Kim (2007), Sung (2009 and 2011) as well as Xing et al. (2009). We also try to put our results in a more general context by showing where they may touch the sphere of interest of other authors.
\end{abstract}

\section{Introduction}

There exist several versions available in the literature for independent sequences of random variables with assumptions of uniform bound or some, quite relaxed, control on their (centered or non-centered) moments. If the independent case is classical in literature, the treatment of dependent variables is more recent. The extension of dependent variable was first discussed considering martingale or different mixing conditions. For instance, Hoeffding [8] and Azuma [2] proved some exponential inequalities for bounded martingale difference sequences. The first author gave the more general exponential inequalities for martingales in $[12,13]$, and Miao et al. [14] proved the deviation inequalities for the linear parameter in stochastic processes by some martingale exponential inequalities.

One of the dependence structure that has attracted the interest of probabilists and statisticians is negatively associated, which is first introduced by Alam and Saxena [1], and carefully studied by Joag-Dev and Proschan [9] and Block et al.

Received by the editors in June 2012 - In revised form in August 2014.

Communicated by T. Bruss.

2010 Mathematics Subject Classification : 60F15, 62G20.

Key words and phrases : Negatively associated, exponential inequalities. 
[3]. A finite family of random variables $\left\{X_{i} ; 1 \leq i \leq n\right\}$ is said to be negatively associated (NA) if for every pair of disjoint subsets $A_{1}$ and $A_{2}$ of $\{1,2, \ldots, n\}$,

$$
\operatorname{Cov}\left\{f_{1}\left(X_{i}, i \in A_{1}\right), f_{2}\left(X_{j}, j \in A_{2}\right)\right\} \leq 0
$$

where $f_{1}$ and $f_{2}$ are coordinatewise increasing and the covariance exists. An infinite family is negatively associated if every finite subfamily is negatively associated. Accordingly, a finite family of random variables $\left\{X_{i} ; 1 \leq i \leq n\right\}$ is said to be positively associated (PA) if for every pair of disjoint subsets $A_{1}$ and $A_{2}$ of $\{1,2, \ldots, n\}$,

$$
\operatorname{Cov}\left\{f_{1}\left(X_{i}, i \in A_{1}\right), f_{2}\left(X_{j}, j \in A_{2}\right)\right\} \geq 0
$$

where $f_{1}$ and $f_{2}$ are coordinatewise increasing and the covariance exists. An infinite family is positively associated if every finite subfamily is positively associated. Similar definition is given in Esary et al. [7] and the interested reader can refer to Roussas [20].

In some applications, negatively associated random variables can be created by different methods (see Joag-Dev and Proschan [9]). Joag-Dev and Proschan [9] showed that many well known multivariate distributions possess the negatively associated property. Some examples include: (a) multinomial, (b) convolution of unlike multinomial, (c) multivariate hypergeometric, (d) Dirichlet, (e) Dirichlet compound multinomial, (f) negatively correlated normal distribution, (g) permutation distribution, (h) random sampling without replacement, and (i) joint distribution of ranks. Also, it occurs in a substantial number of statistical models, multivariate statistical analysis and system reliability. Its significance may have some evidence that negative association is a suitable model for situations, where several species compete for the same limited resources (see Brindley and Thompson [4]). Because of its wide applications, the notion of negative association has received considerable attention recently. For the further fundamental properties and examples of negatively associated sequences, one can refer to Joag-Dev and Proschan [9] and Block et al. [3]. Moreover, for the negatively associated sequence, Newman [15] established the central limit theorem, Matuła [11] studied the three series theorems; Shao and $\mathrm{Su}$ [22] obtained the law of the iterated logarithm; Jing and Liang [17] gave the strong limit theorems for weighted sums.

The exponential inequalities and moment inequalities for partial sum $\sum_{i=1}^{n}\left(X_{i}-E X_{i}\right)$ play a very important role in various proofs of limit theorems. In particular, it provides a measure of convergence rate for the strong law of large numbers. Roussas [19] presented a review of the Bennett's and Hoeffding's inequalities in order to identify the point where independence is actually used, and applies to the negatively associated random variables. Matuła [11] first obtained the Kolmogorov type inequality for the negatively associated random variables. Su et al. [23] studied the moment inequality for negatively associated sequence. Shao [21] proved a comparison theorem on maximal inequalities between negatively associated and independent random variables, and obtained the Rosenthal-type maximal inequality and the Kolmogorov exponential inequality. Sung $[25,26]$ proved some exponential inequalities for negatively associated random variables. Kim and Kim [10] gave a Bernstein-Hoeffding type inequality for strictly stationary and negatively associated random variables. Under mild 
conditions, Jabbari Nooghabi and Azarnoosh [16] got a Bernstein-Hoeffding-type inequality which was established for covariance invariant negatively associated random variables by using a truncation technique together with a block decomposition of the sums to allow an approximation to independence. Xing et al. [27] established an exponential inequality for the negatively associated random variables which improves the corresponding result which was obtained in [10].

In the present paper, we show an exponential inequality for the negatively associated random variables which improves some known results, such as, Kim and Kim [10], Sung [25, 26], Xing et al. [27]. In the next section, we state our main results and give some remarks to compare the known works. The proofs of the results will be obtained in the last section.

\section{Main results}

Throughout the present paper, let $\left\{X_{i}, 1 \leq i \leq n\right\}$ be a sequence of negatively associated identically distributed random variables satisfying the following condition: there exists a constant $\delta>0$, such that

$$
E e^{\delta\left|X_{1}\right|}<\infty
$$

The condition (2.1) is also called exponential integrability condition or Cramér condition, which is often used to establish the exponential convergence rate (e.g., large deviation and moderate deviation principles) or exponential inequalities for the partial sums. In addition, the condition (2.1) is weaker than the assumption of bounded random variables.

For the formulation of the assumptions to be made in this paper, some notations are required. Let $c$ denote a positive constant and for any $1 \leq i \leq n$, define

$$
\begin{aligned}
& X_{1, i}=-c 1_{(-\infty,-c)}\left(X_{i}\right)+X_{i} 1_{[-c, c]}\left(X_{i}\right)+c 1_{(c,+\infty)}\left(X_{i}\right), \\
& X_{2, i}=\left(X_{i}-c\right) 1_{(c,+\infty)}\left(X_{i}\right), \\
& X_{3, i}=\left(X_{i}+c\right) 1_{(-\infty,-c)}\left(X_{i}\right),
\end{aligned}
$$

where $1_{A}$ represents the indicator function of the set $A$. It is easy to check that

$$
X_{i}=X_{1, i}+X_{2, i}+X_{3, i}
$$

for all $1 \leq i \leq n$ and $\left|X_{1, i}\right| \leq c$. It is not difficult to see that if $\left\{X_{n} ; n \geq 1\right\}$ are negatively associated random variables, then $\left\{X_{k, i} ; 1 \leq i \leq n\right\}, n \geq 1, k=1,2,3$, are also negatively associated random variables. 


\subsection{Main results}

The following theorem is our main result in the paper.

Theorem 1. Let $\left\{X_{i}, i \geq 1\right\}$ be a sequence of negatively associated identically distributed random variables satisfying the condition (2.1). Then for any $r>0$,

$$
\begin{aligned}
& P\left(\frac{1}{n} \sum_{i=1}^{n}\left(X_{i}-E X_{i}\right) \geq 3 r\right) \\
\leq & \exp \left\{-\frac{r^{2} n}{2 c^{2}}\right\}+\exp \left\{-\frac{n r^{2} \delta^{2} e^{\frac{1}{2} c \delta}}{4 \sqrt{6} E e^{\delta\left|X_{1}\right|}}\right\}+\exp \left\{-\frac{r^{2} n \delta^{2} e^{\delta c}}{4 E e^{\delta\left|X_{1}\right|}}\right\} \\
\leq & \exp \left\{-\frac{r^{2} n}{2 c^{2}}\right\}+2 \exp \left\{-\frac{n r^{2} \delta^{2} e^{\frac{1}{2} c \delta}}{4 \sqrt{6} E e^{\delta\left|X_{1}\right|}}\right\} \leq 3 e^{-n r^{2} M_{c}}
\end{aligned}
$$

and

$$
\begin{aligned}
& P\left(\frac{1}{n} \sum_{i=1}^{n}\left(X_{i}-E X_{i}\right) \leq-3 r\right) \\
\leq & \exp \left\{-\frac{r^{2} n}{2 c^{2}}\right\}+\exp \left\{-\frac{n r^{2} \delta^{2} e^{\frac{1}{2} c \delta}}{4 \sqrt{6} E e^{\delta\left|X_{1}\right|}}\right\}+\exp \left\{-\frac{r^{2} n \delta^{2} e^{\delta c}}{4 E e^{\delta\left|X_{1}\right|}}\right\} \\
\leq & \exp \left\{-\frac{r^{2} n}{2 c^{2}}\right\}+2 \exp \left\{-\frac{n r^{2} \delta^{2} e^{\frac{1}{2} c \delta}}{4 \sqrt{6} E e^{\delta\left|X_{1}\right|}}\right\} \leq 3 e^{-n r^{2} M_{c}}
\end{aligned}
$$

where

$$
M_{c}=\min \left\{\frac{\delta^{2} e^{\frac{1}{2} c \delta}}{4 \sqrt{6} E e^{\delta\left|X_{1}\right|}}, \frac{1}{2 c^{2}}\right\},
$$

and

$$
0<c \leq \frac{2}{\delta} \log \left(\frac{\sqrt{6} n}{r \delta} E e^{\delta\left|X_{1}\right|}\right) .
$$

From Theorem 1, it is not difficult to obtain the following

Corollary 1. Let $\left\{X_{i}, i \geq 1\right\}$ be a sequence of negatively associated identically distributed random variables satisfying the condition (2.1). Then we have

$$
\frac{\sum_{i=1}^{n}\left(X_{i}-E X_{i}\right)}{\sqrt{n} \log ^{\alpha} n} \stackrel{\text { a.s. }}{\longrightarrow} 0, \text { for all } \alpha>\frac{1}{2} \text {. }
$$

Proof. From Theorem 1 and the Borel-Cantelli lemma, the desired result can be obtained easily. 


\subsection{Some remarks}

In this subsection, we give some remarks to compare with some known works.

Remark 1. Xing et al. [27] gave the following exponential inequality for strictly stationary negatively associated sequence $\left\{X_{i}, i \geq 1\right\}$, which improved the corresponding result which was obtained in Kim and Kim [10]:

$$
P\left(\frac{1}{n}\left|\sum_{i=1}^{n}\left(X_{i}-E X_{i}\right)\right| \geq 3 \varepsilon\right) \leq\left(4+\frac{C M_{\omega}}{4 \alpha^{3} \log ^{3} n}\right) \exp (-\alpha \log n),
$$

where $C, M_{\omega}, \alpha$ are three constants and

$$
\varepsilon=4 \sqrt{\left(\alpha C \log ^{3} n\right) / n} .
$$

However, by using the inequalities in Theorem 1, it is easy to check that we can get a lower-upper bound.

Remark 2. Sung [25] obtained the following result for identically distributed negatively associated random variables. Let $\epsilon_{n}=\sqrt{2 \delta e E\left|X_{1}\right|^{2} c_{n} / n}$, where $\left\{c_{n}, n \geq 1\right\}$ is a sequence of positive numbers such that

$$
0<c_{n}<\left(\frac{e E\left|X_{1}\right|^{2} n}{8 \delta}\right)^{1 / 3}
$$

Then

$$
P\left(\frac{1}{n}\left|\sum_{i=1}^{n}\left(X_{i}-E X_{i}\right)\right|>3 \epsilon_{n}\right) \leq 2\left(1+\frac{E e^{\delta\left|X_{1}\right|}}{\delta^{3} e E\left|X_{1}\right|^{2} c_{n}}\right) e^{-\delta c_{n}} .
$$

The above inequality improved and extended several known results, such as, Kim and Kim [10], Nooghabi and Azarnoosh [16] and Xing et al. [27]. But, Sung's bound can't give an estimation for other $\epsilon_{n}{ }^{\prime}$, for instance, $\epsilon_{n}=\frac{1}{\sqrt{\sqrt{n} \log n}}$, because of the condition (2.5). However, by Theorem 1, we know that

$$
c \sim \log n, \quad M_{c}=\frac{1}{2 c^{2}}
$$

and

$$
P\left(\frac{1}{n}\left|\sum_{i=1}^{n}\left(X_{i}-E X_{i}\right)\right|>3 \epsilon_{n}\right) \leq d_{n} \sim \exp \left\{-\frac{\sqrt{n}}{(\log n)^{3}}\right\} .
$$

Remark 3. From the proof of Theorem 1, we know that if the condition (2.1) is changed into the following: there exists a constant $\delta>0$, such that

$$
\sup _{1 \leq i \leq n} E e^{\delta\left|X_{i}\right|}<\infty,
$$

then the assumption "identically distributed" can be gotten rid off and some small changes in our result could be founded. 
Remark 4. Recently, Sung [26] established several exponential inequalities for negatively dependent random variables (negatively associated random variables are also negatively dependent) with the finite Laplace transforms, which improve on some known results. By comparing with the works in Sung [26], Theorem 1 gives several better bounds than Sung's in some cases.

For an unbounded sequence $\left\{r=r_{n}, n \geq 1\right\}$, Sung obtained the following result (see [26, Theorem 3.5]), for any sequence $\left\{r=r_{n}, n \geq 1\right\}$ of positive real numbers,

$$
P\left(\frac{1}{n}\left|\sum_{i=1}^{n}\left(X_{i}-E X_{i}\right)\right|>r_{n}\right) \leq 2 \exp \left(-\frac{\left(2 r_{n}-K \delta\right) \delta n}{4}\right)
$$

where $K=2\left(E\left|X_{1}\right|^{4}\right)^{1 / 2} E e^{\delta\left|X_{1}\right|}$. If we choose $r_{n}=o(n)$ and $1 / r_{n}=o(1)$ in Theorem 1 , the bound in Theorem 1 is better than the bound in (2.8) for all sufficiently large $n$.

For the bounded sequence $\left\{r=r_{n}, n \geq 1\right\}$, Sung got the following main result (see [26, Theorem 3.1]). Let $\left\{r=r_{n}, n \geq 1\right\}$ and $\left\{c=c_{n}, n \geq 1\right\}$ be sequences of positive real numbers satisfying the following conditions:

(i) $r c \leq e E X_{1}^{2} /(2(3-\gamma))$ for some $0<\gamma<3$,

(ii) $r e^{\delta c / 2} \leq 8 \sqrt{6}\left(E e^{\delta\left|X_{1}\right|}\right)^{3 / 2} /(\gamma \delta)$.

Then

$$
\begin{aligned}
& P\left(\frac{1}{n}\left|\sum_{i=1}^{n}\left(X_{i}-E X_{i}\right)\right| \geq 3 r\right) \\
\leq & 2 \exp \left(-\frac{(3-\gamma)^{2} n r^{2}}{2 e E X_{1}^{2}}\right)+4 \exp \left(-\frac{\gamma^{2} n r^{2} \delta^{2} e^{\delta c / 2}}{64 \sqrt{6}\left(E e^{\delta\left|X_{1}\right|}\right)^{3 / 2}}\right) .
\end{aligned}
$$

If we take $c=\sqrt{e E X_{1}^{2}} /[4(3-\gamma)]$, then for any $r>0$ satisfying

$$
r \leq \min \left\{\sqrt{e E X_{1}^{2}}, 8 e^{\delta c / 2} \sqrt{6}\left(E e^{\delta\left|X_{1}\right|}\right)^{3 / 2} /(\gamma \delta)\right\}
$$

we have

$$
\frac{r^{2} n}{2 c^{2}}>\frac{(3-\gamma)^{2} n r^{2}}{2 e E X_{1}^{2}}
$$

and

$$
\frac{n r^{2} \delta^{2} e^{\frac{1}{2} c \delta}}{4 \sqrt{6} E e^{\delta\left|X_{1}\right|}}>\frac{\gamma^{2} n r^{2} \delta^{2} e^{\delta c / 2}}{64 \sqrt{6}\left(E e^{\delta\left|X_{1}\right|}\right)^{3 / 2}}
$$

where we used $0<\gamma<3$. Hence in this case, the bounds in Theorem 1 are better than Sung's.

Remark 5. In the present paper, we discuss mainly the exponential inequality for the negatively associated random variables. Naturally, there are some papers to study the exponential inequality for the positively associated random variables, such as, Oliveira [18], Sung [24] and so on. We only mention here that positive association is also a typical feature of some sequential selection problems and 
thus attracts interest in problems of optimal stopping. For a recent survey see e.g. Dendievel [5]. The main methods to study the exponential inequality for negatively or positively random variables are to deal with the exponential moment of partial sums. For negatively associated random variables, the exponential moment of partial sums can be controlled by the product of exponential moments (see [9]), and for positively associated random variables, the difference between the exponential moment of partial sums and the product of exponential moments can be controlled by the sums of the covariance (see [6]). Hence the research of positively associated random variables is often more complex than negatively associated sequence.

\section{Proofs of Theorem 1}

Theorem 1 can be proved by the following lemmas.

Lemma 1. [9] Let $A_{1}, \ldots, A_{m}$ be disjoint subsets of $\{1, \ldots, n\}$ and $f_{1}, f_{2}, \ldots, f_{m}$ be increasing positive functions. Then the negatively associated random variables $X_{1}, \cdots, X_{n}$ imply that

$$
E \prod_{i=1}^{m} f_{i}\left(X_{j}, j \in A_{i}\right) \leq \prod_{i=1}^{m} E f_{i}\left(X_{j}, j \in A_{i}\right) .
$$

In particular, for any $\lambda>0$,

$$
E \exp \left\{\lambda \sum_{i=1}^{n} X_{i}\right\} \leq \prod_{i=1}^{n} E e^{\lambda X_{i}}
$$

Lemma 2. [8] Let $X$ be a random variable with $a \leq X \leq b$, where $a<b$ are two constants. Then for any $\lambda>0$,

$$
E \exp \{\lambda(X-E X)\} \leq \exp \left\{\frac{\lambda^{2}}{8}(b-a)^{2}\right\} .
$$

Lemma 3. Let $\left\{X_{i}, i \geq 1\right\}$ be a sequence of negatively associated identically distributed random variables. Then for any $\lambda>0$,

$$
E \exp \left\{\lambda \sum_{i=1}^{n}\left(X_{1, i}-E X_{1, i}\right)\right\} \leq \exp \left\{\frac{1}{2} n \lambda^{2} c^{2}\right\} \text {. }
$$

In particular, for any $r>0$,

$$
P\left(\sum_{i=1}^{n}\left(X_{1, i}-E X_{1, i}\right) \geq r\right) \leq \exp \left\{-\frac{r^{2}}{2 n c^{2}}\right\}
$$

and

$$
P\left(\sum_{i=1}^{n}\left(X_{1, i}-E X_{1, i}\right) \leq-r\right) \leq \exp \left\{-\frac{r^{2}}{2 n c^{2}}\right\}
$$


Proof. From the definition of $X_{1, i}$, it follows that $-c \leq X_{1, i} \leq c$. By Lemma 1 and Lemma 2 , the desired results can be obtained.

Lemma 4. Let $\left\{X_{i}, i \geq 1\right\}$ be a sequence of negatively associated identically distributed random variables satisfying the condition (2.1). Then for any $r>0$ and any $c$ satisfying

$$
0<c \leq \frac{2}{\delta} \log \left(\frac{\sqrt{6} n}{r \delta} E e^{\delta\left|X_{1}\right|}\right)
$$

we have

$$
P\left(\sum_{i=1}^{n}\left(X_{2, i}-E X_{2, i}\right) \geq r\right) \leq \exp \left\{-\frac{r^{2} \delta^{2} e^{\frac{1}{2} c \delta}}{4 \sqrt{6} n E e^{\delta\left|X_{1}\right|}}\right\}
$$

and

$$
P\left(\sum_{i=1}^{n}\left(X_{2, i}-E X_{2, i}\right) \leq-r\right) \leq \exp \left\{-\frac{r^{2} \delta^{2} e^{\delta c}}{4 n E e^{\delta\left|X_{1}\right|}}\right\}
$$

Proof. For any $1 \leq i \leq n$, recall that $X_{2, i}=\left(X_{i}-c\right) 1_{(c,+\infty)}\left(X_{i}\right)$, then it follows that $X_{2, i} \geq 0$ and, by Markov's inequality and Lemma 1 , for any $r>0$ and $\lambda>0$, such that $2 \lambda \leq \delta$, then

$$
\begin{aligned}
P\left(\sum_{i=1}^{n}\left(X_{2, i}-E X_{2, i}\right) \geq r\right) & \leq e^{-\lambda r-n \lambda E X_{2,1} E e^{\lambda \sum_{i=1}^{n} X_{2, i}}} \\
& \leq e^{-\lambda r-n \lambda E X_{2,1}}\left(E e^{\lambda X_{2,1}}\right)^{n}
\end{aligned}
$$

Furthermore, from the following elementary inequality

$$
e^{x} \leq 1+x+\frac{x^{2}}{2} e^{|x|}, \quad \forall x \in \mathbb{R},
$$

and Hölder's inequality, we have

$$
\begin{aligned}
E \exp \left\{\lambda X_{2,1}\right\} & \leq 1+\lambda E X_{2,1}+\frac{\lambda^{2}}{2} E\left(X_{2,1}^{2} e^{\lambda X_{2,1}}\right) \\
& \leq 1+\lambda E X_{2,1}+\frac{\lambda^{2}}{2}\left(E X_{2,1}^{4}\right)^{1 / 2}\left(E e^{2 \lambda X_{2,1}}\right)^{1 / 2}
\end{aligned}
$$

Since $2 \lambda \leq \delta$, then

$$
E e^{2 \lambda X_{2,1}} \leq E e^{\delta\left|X_{1}\right|}
$$

and

$$
\begin{aligned}
E X_{2,1}^{4} & =4 \int_{0}^{\infty} P\left(X_{1}>x+c\right) x^{3} d x \\
& \leq 4 e^{-c \delta} E e^{\delta\left|X_{1}\right|} \int_{0}^{\infty} e^{-\delta x} x^{3} d x=\frac{24}{\delta^{4}} e^{-c \delta} E e^{\delta\left|X_{1}\right|}
\end{aligned}
$$


Hence, by the inequality $1+x \leq e^{x}, \forall x \in \mathbb{R}$, we have

$$
\begin{aligned}
& P\left(\sum_{i=1}^{n}\left(X_{2, i}-E X_{2, i}\right) \geq r\right) \\
\leq & \exp \left\{-\lambda r+n\left(\frac { \lambda ^ { 2 } } { 2 } ( E X _ { 2 , 1 } ^ { 4 } ) ^ { 1 / 2 } \left(E e^{\left.\left.\left.2 \lambda X_{2,1}\right)^{1 / 2}\right)\right\}}\right.\right.\right. \\
\leq & \exp \left\{-\lambda r+n\left(\lambda^{2} \frac{\sqrt{6}}{\delta^{2}} e^{-\frac{1}{2} c \delta} E e^{\delta\left|X_{1}\right|}\right)\right\} \\
\leq & \exp \left(-\frac{r^{2} \delta^{2} e^{\frac{1}{2} c \delta}}{4 \sqrt{6} n E e^{\delta\left|X_{1}\right|}}\right),
\end{aligned}
$$

by taking

$$
\lambda=\frac{r \delta^{2} e^{\frac{1}{2} c \delta}}{2 \sqrt{6} n E e^{\delta\left|X_{1}\right|}}
$$

Here, from

$$
c \leq \frac{2}{\delta} \log \left(\frac{\sqrt{6} n}{r \delta} E e^{\delta\left|X_{1}\right|}\right),
$$

it is easy to check that $2 \lambda \leq \delta$.

Next we discuss the inequality (3.4). Note that $-X_{2, i} \leq 0$, then for any $\lambda>0$, by the elementary inequalities,

$$
e^{-x} \leq 1-x+\frac{1}{2} x^{2}, \quad \forall x \geq 0 \text { and } 1+x \leq e^{x}, \forall x \in \mathbb{R},
$$

we have, from Lemma 1,

$$
\begin{aligned}
& P\left(\sum_{i=1}^{n}\left(X_{2, i}-E X_{2, i}\right) \leq-r\right) \\
\leq & e^{-\lambda r} \prod_{i=1}^{n} e^{\lambda E X_{2, i}} E\left(e^{-\lambda X_{2, i}}\right) \\
\leq & e^{-\lambda r} \prod_{i=1}^{n} e^{\lambda E X_{2, i}}\left(1-\lambda E X_{2, i}+\frac{\lambda^{2}}{2} E X_{2, i}^{2}\right) \\
\leq & \exp \left(-\lambda r+\frac{\lambda^{2}}{2} \sum_{i=1}^{n} E X_{2, i}^{2}\right) \\
= & \exp \left(-\lambda r+\frac{\lambda^{2}}{2} n E X_{2,1}^{2}\right), \text { for all } \lambda>0 .
\end{aligned}
$$

In particular, for $\lambda=\frac{r}{n E X_{2,1}^{2}}$, we get

$$
P\left(\sum_{i=1}^{n}\left(X_{2, i}-E X_{2, i}\right) \leq-r\right) \leq \exp \left(-\frac{r^{2}}{2 n E X_{2,1}^{2}}\right)
$$


Since

$$
\begin{aligned}
E X_{2,1}^{2} & =2 \int_{0}^{\infty} t P\left(X_{1}>c+t\right) d t \\
& \leq 2 e^{-\delta c} E e^{\delta\left|X_{1}\right|} \int_{0}^{\infty} t e^{-\delta t} d t=\frac{2 e^{-\delta c} E e^{\delta\left|X_{1}\right|}}{\delta^{2}}
\end{aligned}
$$

then it follows that

$$
P\left(\sum_{i=1}^{n}\left(X_{2, i}-E X_{2, i}\right) \leq-r\right) \leq \exp \left\{-\frac{r^{2} \delta^{2} e^{\delta c}}{4 n E e^{\delta\left|X_{1}\right|}}\right\} .
$$

Lemma 5. Let $\left\{X_{i}, i \geq 1\right\}$ be a sequence of negatively associated identically distributed random variables satisfying the condition (2.1). Then for any $r>0$ and any $c$ satisfying

$$
0<c \leq \frac{2}{\delta} \log \left(\frac{\sqrt{6} n}{r \delta} E e^{\delta\left|X_{1}\right|}\right)
$$

we have

$$
P\left(\sum_{i=1}^{n}\left(X_{3, i}-E X_{3, i}\right) \leq-r\right) \leq \exp \left\{-\frac{r^{2} \delta^{2} e^{\frac{1}{2} c \delta}}{4 \sqrt{6} n E e^{\delta\left|X_{1}\right|}}\right\}
$$

and

$$
P\left(\sum_{i=1}^{n}\left(X_{3, i}-E X_{3, i}\right) \geq r\right) \leq \exp \left\{-\frac{r^{2} \delta^{2} e^{\delta c}}{4 n E e^{\delta\left|X_{1}\right|}}\right\} .
$$

Proof. As the same proof as Lemma 4, the desired results in the lemma can be obtained.

Proof of Theorem 1. By Lemmas 3-5 and the following inequality

$$
\frac{\delta^{2} e^{\frac{1}{2} c \delta}}{4 \sqrt{6} E e^{\delta\left|X_{1}\right|}}<\frac{\delta^{2} e^{\delta c}}{4 E e^{\delta\left|X_{1}\right|}}
$$

for any $r>0$, we have

$$
\begin{aligned}
& P\left(\frac{1}{n} \sum_{i=1}^{n}\left(X_{i}-E X_{i}\right) \geq 3 r\right) \\
\leq & \exp \left\{-\frac{r^{2} n}{8 c^{2}}\right\}+\exp \left\{-\frac{n r^{2} \delta^{2} e^{\frac{1}{2} c \delta}}{4 \sqrt{6} E e^{\delta\left|X_{1}\right|}}\right\}+\exp \left\{-\frac{r^{2} n \delta^{2} e^{\delta c}}{4 E e^{\delta\left|X_{1}\right|}}\right\} \\
\leq & 3 e^{-n r^{2} M_{c}}
\end{aligned}
$$

where

$$
M_{c}=\min \left\{\frac{\delta^{2} e^{\frac{1}{2} c \delta}}{4 \sqrt{6} E e^{\delta\left|X_{1}\right|}}, \frac{1}{8 c^{2}}\right\} .
$$

The inequality (2.4) can be obtained by a similar argument. 


\section{Acknowledgements}

The authors are very grateful to the referees for their valuable reports which improved the presentation of this work. This work is supported by IRTSTHN (14IRTSTHN023), NSFC (11001077, 11471104), NCET (NCET-11-0945), and Plan For Scientific Innovation Talent of Henan Province (124100510014).

\section{References}

[1] K. Alam and K. M. L. Saxena, Positive dependent in multivariate distributions. Communications in Statistics-Theory and Methods, 1981, 10: 1183-1196.

[2] K. Azuma, Weighted sums of certain dependent random variables. Tohoku Mathematical Journal, 1967, 19: 357-367.

[3] H. M. Block, T. H. Savits and M. Sharked, Some concepts of negative dependence. Annals of Probability, 1982, 10: 765-772.

[4] E. C. Brindley Jr. and W. A. Thompson Jr., Dependence and aging aspects of multivariate survival. J. Amer. Statist. Assoc., 1972, 67: 822-830.

[5] R. Dendievel, New developments of the odds-theorem. Math. Sci., 2013, 38(2): 111-123.

[6] I. Dewan and B. L. S. Prakasa Rao, A general method of density estimation for associated random variables. J. Nonparametric Statist., 1999, 10: 405-420.

[7] J. D. Esary, F. Proschan and D. W. Walkup, Association of random variables with applications. Ann. Math. Statist., 1967, 38: 1466-1474.

[8] W. Hoeffding, Probability inequalities for sums of bounded random variables. Journal of the American Statistical Association, 1963, 58: 13-30.

[9] K. Joag-Dev and F. Proschan, Negative association of random variables with applications. Annals of Statistics, 1983, 11: 286-295.

[10] T. S. Kim and H. C. Kim, On the exponential inequality for negatively dependent sequence. Communications of the Korean Mathematical Society, 2007, 22(2): 315-321.

[11] P. Matuła, A note on the almost sure convergence of sums of negatively dependent random variables. Statistics \& Probability Letters, 1992, 15(3): 209-213.

[12] Y. Miao, A note on the martingale inequality. Journal of Inequalities in Pure and Applied Mathematics, 2006, 7(5): Article 187.

[13] Y. Miao, Concentration inequalities for semi-bounded martingales. ESAIM: Probability and Statistics, 2008, 12: 51-57.

[14] Y. Miao, Y. W. Jiang and S. Shen, Deviation probability bound for linear parameter in stochastic processes. Communications in Statistics-Theory and Methods, 2009, 38(6): 888-901. 
[15] C. M. Newman, Asymptotic independence and limit theorems for positively and negatively dependent random variables. Inequalities in statistics and probability (Lincoln, Neb., 1982), 127-140, IMS Lecture Notes Monogr. Ser., 5, Inst. Math. Statist., Hayward, CA, 1984.

[16] H. Jabbari Nooghabi and H. A. Azarnoosh, Exponential inequality for negatively associated random variables. Statistical Papers, 2009, 50(2): 419-428.

[17] B. Y. Jing and H. Y. Liang, Strong limit theorems for weighted sums of negatively associated random variables. J. Theoret. Probab. 21, no. 4, 890-909 (2008).

[18] P. D. Oliveira, An exponential inequality for associated variables. Statistics \& Probability Letters, 2005, 73: 189-197.

[19] G. G. Roussas, Exponential probability inequalities with some applications. IMS Lecture Notes-Monograph Series, 1996, 30: 303-319.

[20] G. G. Roussas, Positive and negative dependence with some statistical application. In: Ghosh, S. (Ed.), Asymptotics Nonparametrics and Time Series. Marcel Dekker, New York, 1999, 757-788.

[21] Q. M. Shao, A comparison theorem on maximal inequalities between negatively associated and independent random variables. Journal of Theoretical Probability, 2000, 13(2): 343-356.

[22] Q. M. Shao and C. Su, The law of the iterated logarithm for negatively associated random variables. Stochastic Process. Appl. 83, no. 1, 139-148. (1999).

[23] C. Su, L. C. Zhao and Y. B. Wang, Moment inequalities and weak convergence for negatively associated sequences. Sci. China Ser. A 40, no. 2, 172-182 (1997).

[24] S. H. Sung, A note on the exponential inequality for associated random variables. Statistics \& Probability Letters, 2007, 77: 1730-1736.

[25] S. H. Sung, An exponential inequality for negatively associated random variables. Journal of Inequalities and Applications, 2009, Article 649427.

[26] S. H. Sung, On the exponential inequalities for negatively dependent random variables. Journal of Mathematical Analysis and Applications, 2011, 381(2): 538-545.

[27] G. Xing, S. Yang, A. Liu, and X. Wang, A remark on the exponential inequality for negatively associated random variables. Journal of the Korean Statistical Society, 2009, 38(1): 53-57.

College of Mathematics and Information Science,

Henan Normal University,

Henan Province, 453007, China.

email:yumiao728@yahoo.com.cn, jianyongmu@163.com 